\title{
COLLISIONAL LINE SHAPES IN THE PRESENCE OF BROAD-BAND SQUEEZED VACUUM
}

\author{
N.H. Cong AND L.V. VINH \\ Department of Physics, University of Vinh \\ 182 Le Duan Str., Vinh City, Vietnam
}

(Received October 9, 2000; in final form February 12, 2001)

\begin{abstract}
In this paper we discuss the line shapes of a two-level atom in the presence of broad-band squeezed vacuum and collisional broadening. We describe the collisions and the squeezed vacuum using a stochastic model of Rabi amplitude fluctuations. From such a stochastic description the fast and the slow damping rates of the Bloch polarizations are obtained. The strong field resonance fluorescence spectrum in the presence of stochastic broad-band squeezed vacuum and collisions is derived.
\end{abstract}

PACS numbers: 42.50 .Lc, $42.50 . \mathrm{Ct}$

\section{Introduction}

The stochastic Bloch equations (SBE) with multiplicative fluctuations can be written in the form of the following stochastic-matrix-differential equation [1] (repeated indices indicate summation):

$$
\frac{\mathrm{d} V}{\mathrm{~d} t}=\left[M_{0}+\mathrm{i} x_{k}(t) M_{k}\right] V,
$$

where $x_{k}$ are external noises, $M_{0}$ is the deterministic (coherent part) of the Bloch evolution and the matrices $M_{k}$ describe the coupling of the Bloch variables (denoted by $V$ ) to the noise. For a wide class of external noises, stochastic differential Eq. (1) can be averaged and as a result the stochastic expectation value of $V(t)$, denoted here by $\langle V(t)\rangle$, satisfies the following differential equation:

$$
\frac{\mathrm{d}\langle V(t)\rangle}{\mathrm{d} t}=\left(M_{0}-\Sigma\right)\langle V(t)\rangle,
$$

where $\Sigma$ is the relaxation matrix or the line broadening operator (LBO). Depending on the model and the source of the fluctuations, different expressions for the line broadening operator $\Sigma$ can be obtained. In the literature one can find extensive reviews of different theoretical schemes leading to the LBO. 
In the framework of the quantum noise-operator methods the radiative decay of a two-level atom interacting with a broad-band squeezed vacuum has been investigated $[2,3]$. It has been shown that the Bloch polarizations have two different damping rates. One of these polarizations is damped at the enhanced rate and the other at the reduced rate compared to the normal radiative decay. The quantum noise-operator methods have been applied to a wide class of problems involving squeezed light. Resonance fluorescence from an atom in a squeezed vacuum [4] and the inhibition of atomic-phase decays by squeezed light [5] have been investigated.

In a different approach to the quantum noise, stochastic density-matrix equations for an atom strongly driven by a finite-bandwidth squeezed light have been derived $[6,7]$. The authors of [8] have shown that the squeezed vacuum can be incorporated in the form of the multiplicative stochastic equation (1). It is known that a stochastic theory of spontaneous emission without atomic fluctuations will predict spurious effects like spontaneous absorption of the ground state [9]. The atomic fluctuations cancel exactly the vacuum fluctuations of spontaneous absorption, leaving the atom in its ground state [10]. This effect can be incorporated in the SBE if the $c$-number multiplicative stochastic process that represents the vacuum fluctuations drives only the population $p_{2}$ of the excited state and is inhibited due to atomic fluctuations for the population of the ground state $p_{1}$ [11].

In this paper we present an extension of the stochastic method investigating a two-level atom perturbed by stochastic collisions and in the presence of the broad-band squeezed vacuum. This simple statistical model of the collisional relaxations lead to an explicit expression for the line broadening operator. We show that this LBO in the long-time limit can be reduced to two lifetimes $T_{1}$ and $T_{2}$. We illustrate our approach, deriving the fast and the slow damping rates of the Bloch polarizations from a stochastic model of squeezed vacuum fluctuations. The power-spectrum of the strong-field resonance fluorescence in the presence of broad-band squeezed vacuum and collision is derived and discussed.

This paper is organized in the following way. In Section 2 we introduce a stochastic model of collisions based on a random telegraph signal description of the atomic frequency. In Section 3, we derive the essentials of the SBE in the presence of collision and a c-number stochastic vacuum noise and external coherent laser field. In Section 4 we derive the damping matrix for a two-level atom coupled to a stochastic broad-band squeezed vacuum and collisions. Finally, Section 5 contains some concluding remarks.

\section{Stochastic model of collisions}

We are going to assume that the environment of a two-level system acts as a stochastic reservoir and gives rise to fluctuations of the atomic resonant frequency $\omega_{0}$. We shall model the influence of the buffer gas on the emitter assuming that the instantaneous frequency of the two-level atom is $\omega_{0}+x_{c}(t)$, where $x_{c}(t)$ describes the collisional stochastic fluctuations.

In the simplest approach to the stochastic description of the buffer gas reservoir, we shall assume that $x_{c}(t)$ is a random variable described by a two-step random telegraph signal (RTS) jumping between two states $a$ and $-a$. This very 
simple well-known dichotomic Markov process is fully defined by the following correlations:

$$
\left\langle x_{c}(t)\right\rangle=0 \quad \text { and } \quad\left\langle x_{c}(t) x_{c}\left(t^{\prime}\right)\right\rangle=a^{2} \exp \left(-\frac{\left|t-t^{\prime}\right|}{\tau}\right),
$$

where $2 \tau=T$ is the telegraph jump time. The physical picture of these fluctuations is quite simple. If $k$ is the wave vector of the emitted photon and $v$ is the instantaneous velocity of the emitter one can assume that due to collisions only the direction of $k$ changes at random. At each time when the emitter is assumed to undergo collisions with the perturbers the angle between $k$ and $\boldsymbol{v}$ suffers an abrupt change of $\pi$ or $-\pi$. Between collisions the velocity of emitter stays constant and as a result we can write the RTS in the following form $x_{\mathrm{c}}(t)=k v \xi$, where $\xi=(-1)^{n(t)}$. In this expression $n(t)$ is a number of time the telegraph changes its state due to collisions. From microscopic considerations we can associate $\gamma=1 / \tau$ with the rate at which the emitter, with the velocity $v$, suffers the next collision. In such a case

$$
\gamma(v)=\pi n_{\mathrm{p}} r_{0}^{2} v
$$

where $n_{\mathrm{p}}$ is the number of perturbers per unit volume and $r_{0}$ is an effective scattering radius. In this statistical picture of collision the effect of the buffer gas is viewed to be so drastic that the velocity of the emitter after the collision is assumed to be completely independent of its velocity before the collision.

\section{SBE in the presence of stochastic broad-band vacuum and collision}

The atomic Bloch equations driven by a quantum noise are going to be investigated in the framework of c-number multiplicative stochastic Rabi amplitudes. A stochastic model of squeezed vacuum has been introduced and investigated in [8]. It is well known that the broad-band squeezed vacuum generated in optical parametric oscillation is described by two parameters $N$ and $M$, which characterize the reduced quantum fluctuations of the vacuum quadratures [12]. The real parameter $N$ can be associated with a thermal component carried by the broad-band squeezed light, while the complex parameter $M=|M| \exp (\mathrm{i} \varphi)$ represents the phase-dependent vacuum contribution due to the squeezed quantum noise. Guided by this physical interpretation we incorporate broad-band squeezed fluctuations assuming that the squeezed vacuum is described by the following complex random two Rabi amplitudes $\Omega_{\mathrm{t}}$ and $\Omega_{\mathrm{v}}$. The thermal part $\Omega_{\mathrm{t}}=x_{\mathrm{t}}+\mathrm{i} y_{\mathrm{t}}$ couples to the atomic degrees of freedom in the standard way. The vacuum part $\Omega_{\mathrm{v}}=x_{\mathrm{v}}+\mathrm{i} y_{\mathrm{v}}$ couples to the atomic degrees in the different way. We shall assume that the coherent light field $\Omega_{0}=x_{1}+\mathrm{i} y_{1}$ is deterministic.

The SBE in the presence of these random fields are obtained in the following way. The light part of the driving field and collision couples to the three components $(u, v, w)$ of the Bloch vector in the standard way [13], while the vacuum part of the field drives only the population of the excited state and is inhibited due to atomic fluctuations for the population of the ground state [11]. With this basic assumption the SBE in this case have the following form: 


$$
\begin{aligned}
& \dot{u}=-\left(\Delta+x_{\mathrm{c}}(t)\right) v+y_{1} w+\frac{1}{2} y_{\mathrm{v}}(w+1), \\
& \dot{v}=\left(\Delta+x_{\mathrm{c}}(t)\right) u+x_{1} w+\frac{1}{2} x_{\mathrm{v}}(w+1), \\
& \dot{w}=-\left(y_{1}+y_{\mathrm{v}}\right) u-\left(x_{1}+x_{\mathrm{v}}\right) v,
\end{aligned}
$$

where $\Delta$ is the detuning of driving coherent field frequency from the atomic resonance frequency. Note that the vacuum Rabi frequency is coupled only to the population of the excited state $p_{2}=\frac{1}{2}(w+1)$. This takes into account the fact that in the vacuum only spontaneous emission can occur, but never spontaneous absorption.

Fluctuations of the thermal part of the squeezed light are described by a Gaussian white-noise with a mean value equal to zero and the following non-vanishing autocorrelations:

$$
\begin{aligned}
& \left\langle x_{\mathrm{t}}(t) x_{\mathrm{t}}\left(t^{\prime}\right)\right\rangle=2 A N \delta\left(t-t^{\prime}\right), \\
& \left\langle y_{\mathrm{t}}(t) y_{\mathrm{t}}\left(t^{\prime}\right)\right\rangle=2 A N \delta\left(t-t^{\prime}\right),
\end{aligned}
$$

where $A$ is the atomic decay rate for spontaneous emission into the unsqueezed vacuum and where the parameter $N$ characterized the "thermal" contribution of the squeezed vacuum.

Fluctuations of the two vacuum components are described by Gaussian stochastic processes with a mean value equal to zero and the following correlations:

$$
\begin{aligned}
& \left\langle x_{\mathrm{v}}(t) x_{\mathrm{v}}\left(t^{\prime}\right)\right\rangle=2 A(1-2|M| \cos \varphi) \delta\left(t-t^{\prime}\right), \\
& \left\langle y_{\mathrm{v}}(t) y_{\mathrm{v}}\left(t^{\prime}\right)\right\rangle=2 A(1+2|M| \cos \varphi) \delta\left(t-t^{\prime}\right), \\
& \left\langle x_{\mathrm{V}}(t) y_{\mathrm{v}}\left(t^{\prime}\right)\right\rangle=2 A|M| \sin \varphi \delta\left(t-t^{\prime}\right) .
\end{aligned}
$$

Note that the diffusion coefficients are phase-dependent. This reflects the fact that squeezed vacuum fluctuations are described by a stochastic process whose Fokker-Planck equation contains phase-dependent diffusion terms. In general the parameters $N$ and $M$ can be changed independently, though for the ideal squeezing, they are connected by the relation: $|M|=\sqrt{N(N+1)}$. The apparent nonpositive diffusion coefficients for the vacuum component do not cause mathematical problems. Only the entire combined thermal and vacuum field form the squeezed stochastic field [6]. The stochastic differential Eq. (5) driven by the correlated Gaussian noise and RTS can be averaged exactly. The stochastic expectation value of $V(t)$ is the solution of the following integro-differential equation:

$$
\frac{\mathrm{d}\langle V(t)\rangle}{\mathrm{d} t}=\left(M_{0}-\Sigma_{s}\right)\langle V(t)\rangle-\int_{0}^{t} \mathrm{~d} s \Sigma_{e}(t-s)\langle V(s)\rangle,
$$

where $\Sigma_{s}$ and $\Sigma_{e}$ are two parts of the relaxation matrix $\Sigma$ : 


$$
\Sigma_{s}=D_{k l} M_{k} M_{l}
$$

where $D_{k l}$ are various diffusion coefficients of the white noise and the Laplace transform of the line broadening kernel for collisions has the following form:

$$
\Sigma_{c}=a^{2} M_{\mathrm{c}} \frac{1}{z+(1 / \tau)-M_{0}} M_{\mathrm{c}},
$$

where $M_{\mathrm{C}}$ is the matrix of the multiplicative collisional noise. If we focus our attention on long-time behavior which is valid if all early transients have died away, we can perform the well-known "pole approximation" taking $z=0$ in $\Sigma(z)$. In the long-time approximation the integro-differential Eq. (8) reduces to Eq. (2) with the LBO in the following form:

$$
\Sigma=D_{k l} M_{k} M_{l}+a^{2} M_{c} \frac{1}{(1 / \tau)-M_{0}} M_{c} .
$$

From this expression the averaged SBE are

$$
\begin{aligned}
& \langle\dot{u}\rangle=-A\left(N+\frac{1}{2}+|M| \cos \varphi+\Gamma_{u}\right)\langle u\rangle-(A|M| \sin \varphi+\Delta)\langle v\rangle, \\
& \langle\dot{v}\rangle=-A\left(N+\frac{1}{2}-|M| \cos \varphi+\Gamma_{v}\right)\langle v\rangle-(A|M| \sin \varphi-\Delta)\langle u\rangle+\Omega_{0}\langle w\rangle, \\
& \langle\dot{w}\rangle=-A(2 N+1)\langle w\rangle-A-\Omega_{0}\langle v\rangle,
\end{aligned}
$$

where we have assumed for simplicity that the coherent Rabi amplitude $\Omega_{0}$ is real and

$$
\Gamma_{u}=a^{2} \tau, \quad \Gamma_{v}=\frac{a^{2} \tau}{1+\Omega_{0}^{2} \tau^{2}} .
$$

We recognize in these equations the Bloch equations with the enhanced and the reduced damping rates [2]. At exact resonance of the driving field with the atomic transition frequency three different lifetimes can be calculated from the LBO. The transverse matrix damping rate in the $(u, v)$-plane that results from the c-number stochastic squeezed-vacuum fluctuations is phase-dependent and has the following form:

$$
\frac{1}{T_{2}}=A\left(\begin{array}{cc}
N+(1 / 2)+|M| \cos \varphi+\Gamma_{u} & |M| \sin \varphi \\
|M| \sin \varphi & N+(1 / 2)-|M| \cos \varphi+\Gamma_{v}
\end{array}\right) .
$$

The corresponding noise-induced longitudinal damping rate is

$$
\frac{1}{T_{1}}=A(2 N+1)
$$

The transverse matrix damping is off-diagonal. This suggests that the polarization vector can be reduced to "normal modes", leading to diagonal damping rates

$$
\lambda_{1,2}=\frac{A}{2}\left[\left(2 N+1+\Gamma_{u}+\Gamma_{v}\right) \pm g\right]
$$

where

$$
g=
$$

$$
\sqrt{\left(2 N+\frac{1}{2}+\Gamma_{u}+\Gamma_{v}\right)^{2}-4\left(N+\frac{1}{2}+\Gamma_{u}+|M| \cos \varphi\right)\left(N+\frac{1}{2}+\Gamma_{v}-|M| \cos \varphi\right)} .
$$


These two eigenvalues of the damping matrix are associated with dampings of the two "normal modes" being linear combinations of $\langle u\rangle$ and $\langle v\rangle$. Note that in general the damping rates in the presence of collisions depend on the power of driving field. This results in an asymmetry between the transverse lifetimes of the Bloch components $u$ and $v$. This asymmetry between two lifetimes is most pronounced in the limit $\Omega_{0} \gg \tau$, when a driving field with a Rabi amplitude is much larger than the collisional lifetime [14]. In this case we have $\Gamma_{v}=0$ and only the collisional $\Gamma_{u}$ contributes. In the white-noise limit, i.e., when $\tau \rightarrow 0$ with fixed $a^{2} \tau$ we have $\Gamma_{u}=\Gamma_{v}=a^{2} \tau$.

\section{Resonance fluorescence in the presence of the stochastic vacuum and collision}

Because the quantum fluctuations have been incorporated in the SBE in the form of $c$-number random amplitudes, the power-spectrum of a noise-induced dipole correlation describes the resonance fluorescence of a radiating dipole moment in the presence of squeezed vacuum. Correlations between the radiating dipoles are induced by the vacuum noise. The theoretical methods involved in the calculations of stochastically induced resonance fluorescence spectra have been described in great detail elsewhere [15, 16].

For the steady-state autocorrelation of dipole we define the following quantities:

$$
S_{\mathrm{d}}(\omega)=\lim _{t \rightarrow \infty} 2 \operatorname{Re} \int_{0}^{\infty} \exp (-\mathrm{i} \omega \tau)\left\langle d^{*}(t+\tau) d(t)\right\rangle \mathrm{d} \tau .
$$

The quantity represents the stationary spectrum of dipole fluctuation. In order to calculate the dipole-dipole correlations required in the definition (18) of the dipole power spectrum, we introduce the following four-component transposed vector:

$$
V(t, \tau)=\left(d^{*}(t+\tau) d(t), d(t+\tau) d(t), p_{2}(t+\tau) d(t), d(t)\right)
$$

where the complex dipole moment $d$ is defined as $u=d+d^{*}$ and $v=\mathrm{i}\left(d-d^{*}\right)$. By a repeated application of the stochastic Bloch equation (5) it is easy to check that this four-component vector satisfies a differential equation

$$
\begin{aligned}
\frac{\mathrm{d}}{\mathrm{d} \tau} V_{1} & =\frac{\mathrm{i}}{2} x_{\mathrm{c}} V_{1}+\frac{\mathrm{i}}{2}\left(2 \Omega_{0}+\Omega_{\mathrm{v}}^{*}+2 \Omega_{\mathrm{t}}^{*}\right) V_{3}-\frac{\mathrm{i}}{2}\left(\Omega_{0}+\Omega_{\mathrm{t}}^{*}\right) V_{4}, \\
\frac{\mathrm{d}}{\mathrm{d} \tau} V_{2} & =-\frac{\mathrm{i}}{2} x_{\mathrm{c}} V_{2}-\frac{\mathrm{i}}{2}\left(2 \Omega_{0}+\Omega_{\mathrm{v}}+2 \Omega_{\mathrm{t}}\right) V_{3}+\frac{\mathrm{i}}{2}\left(\Omega_{0}+\Omega_{\mathrm{t}}\right) V_{4}, \\
\frac{\mathrm{d}}{\mathrm{d} \tau} V_{3} & =\frac{\mathrm{i}}{2}\left(\Omega_{0}+\Omega_{\mathrm{v}}+\Omega_{\mathrm{t}}\right) V_{1}-\frac{\mathrm{i}}{2}\left(\Omega_{0}+\Omega_{\mathrm{v}}^{*}+\Omega_{\mathrm{t}}^{*}\right) V_{2}, \\
\frac{\mathrm{d}}{\mathrm{d} \tau} V_{4} & =0 .
\end{aligned}
$$

From these equations we conclude that this 4-component vector satisfies a stochastic differential equation in a form given by (1) with time-independent matrices $M_{0}, M_{k}$ and the following initial condition for:

$$
V(t, \tau=0)=\left(d^{*}(t) d(t), d(t) d(t), p_{2}(t) d(t), d(t)\right) .
$$


Performing the average of the multiplicative stochastic equations (20) we obtain the following averaged Bloch equations for second order atomic correlation functions:

$$
\begin{aligned}
& \frac{\mathrm{d}}{\mathrm{d} \tau}\left\langle V_{1}\right\rangle=\left[-\frac{A}{2}(2 N+1)+b_{11}\right]\left\langle V_{1}\right\rangle-\left(A|M| \exp (\mathrm{i} \varphi)+b_{12}\right)\left\langle V_{2}\right\rangle \\
& +\mathrm{i} \Omega_{0}\left\langle V_{3}\right\rangle-\frac{\mathrm{i}}{2} \Omega_{0}\langle d\rangle, \\
& \frac{\mathrm{d}}{\mathrm{d} \tau}\left\langle V_{2}\right\rangle=-\left(A|M| \exp (-\mathrm{i} \varphi)+b_{21}\right)\left\langle V_{1}\right\rangle-\left[\frac{A}{2}(2 N+1)+b_{22}\right]\left\langle V_{2}\right\rangle \\
& -\mathrm{i} \Omega_{0}\left\langle V_{3}\right\rangle+\frac{\mathrm{i}}{2} \Omega_{0}\langle d\rangle, \\
& \frac{\mathrm{d}}{\mathrm{d} \tau}\left\langle V_{3}\right\rangle=\frac{\mathrm{i}}{2} \Omega_{0}\left\langle V_{1}\right\rangle-\frac{\mathrm{i}}{2} \Omega_{0}\left\langle V_{2}\right\rangle-A(2 N+1)\left\langle V_{3}\right\rangle+A N\langle d\rangle .
\end{aligned}
$$

In Eq. (22) we have used the following notations:

$$
b_{11}=b_{22}=\frac{a^{2}\left[(1 / \tau)^{2}+\Omega_{0}^{2} / 2\right]}{P_{a}}, \quad b_{12}=b_{21}=\frac{a^{2} \Omega_{0}^{2}}{P_{a}},
$$

where

$$
P_{a}=\frac{1}{\tau}\left[\left(\frac{1}{\tau}\right)^{2}+\Omega_{0}^{2}\right]
$$

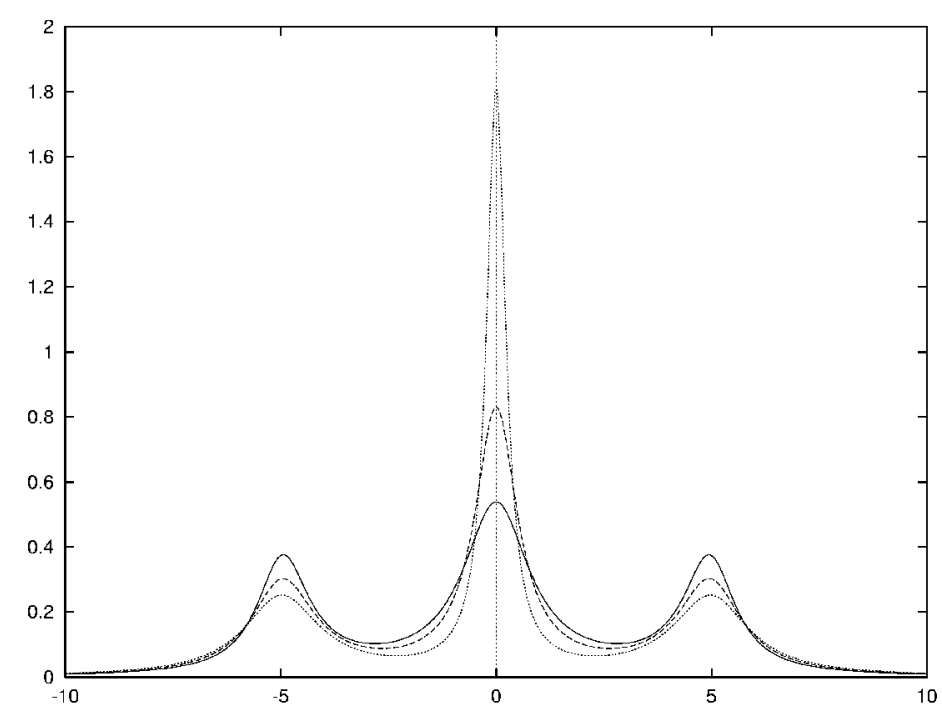

Fig. 1. Resonance fluorescence power-spectrum as a function of the squeezed phase $\varphi$. The solid line corresponds to $\varphi=0$, the dashed line $-\varphi=\pi / 2$, and the dotted line $\varphi=\pi$. Plots are for $A=1, \Omega_{0}=5, N=0.1, a=0$ and $\tau=0.01$. 


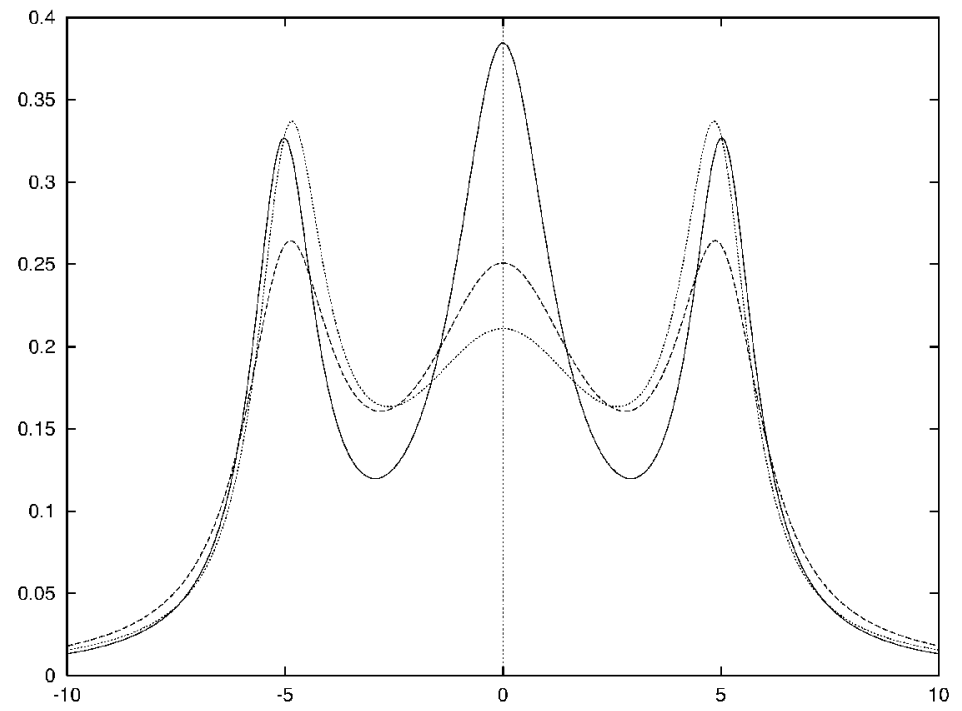

Fig. 2. Resonance fluorescence power-spectrum as a function of the squeezed parameter $N$. The solid line corresponds to $N=0.1$, the dashed line $-N=0.5$, and the dotted line $-N=1$. Plots are for $A=1, \Omega_{0}=5, a=2, \tau=0.1$, and $\varphi=0$.

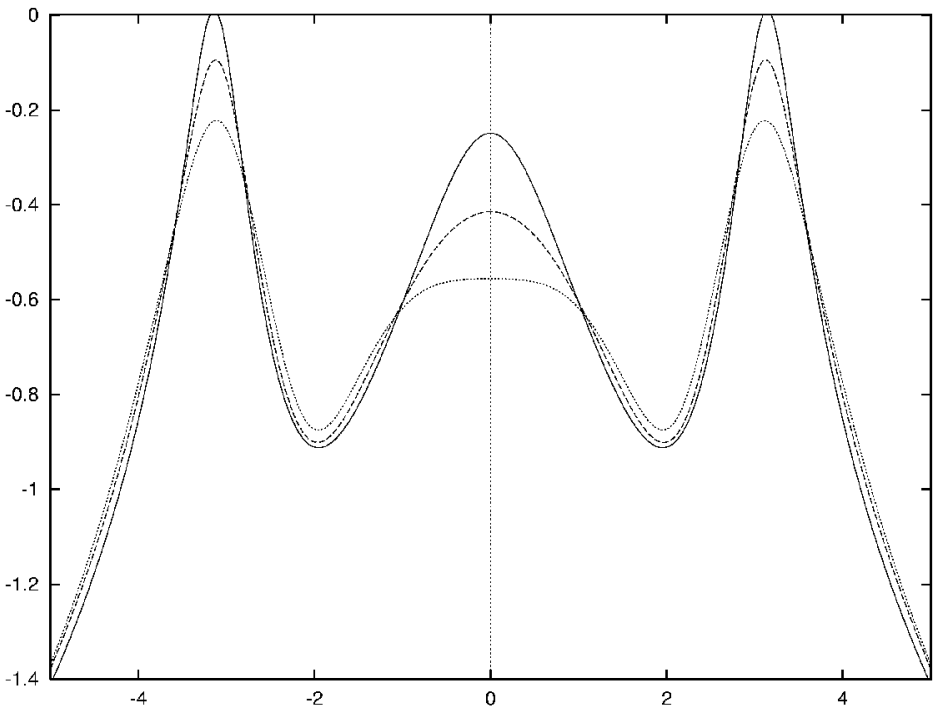

Fig. 3. Resonance fluorescence power-spectrum $\left(\log _{10} S\right)$ as a function of the coherence time $\tau$ of the collisional stochastic fluctuation. The solid line corresponds to $\tau=0.2$, the dashed line $-\tau=0.4$, and the dotted line $-\tau=0.6$. Plots are for $A=0, \Omega_{0}=3$, $a=2, \varphi=0$, and $N=M=0$. 
We solve system of equations (22) for the dipole-dipole correlation function, and we evaluate the power-spectrum using (18). As a result the strong-field resonance fluorescence spectrum in the presence of broad-band squeezed vacuum and collision is given by the following formula:

$$
S(\omega)=\frac{Z(\omega)}{N(\omega)}
$$

where the functions $Z(\omega)$ and $N(\omega)$ are defined in the Appendix.

In Fig. 1 we have plotted the resonance fluorescence spectrum in the presence of a stochastic squeezed vacuum $(N=0.1)$ and collision as a function of $\varphi$. In Fig. 2 we have plotted the resonance fluorescence spectrum in the presence of a stochastic squeezed vacuum and collision with different values of the parameter $N$. In Fig. 3 we have plots of $\log _{10} S$ in the presence of only collision $(N=M=0)$. We note that these spectra have the same form as those in [17].

\section{Conclusions}

In this paper we have discussed characteristics of the strong atom-field interaction in the presence of squeezed vacuum and collision. We have shown that the atomic Bloch equations driven by a quantum noise can be studied in the framework of a stochastic multiplicative equation of the form given by (1) with $c$-number stochastic Rabi amplitudes. We have shown that the squeezed vacuum stochastic fluctuations represented by $c$-number random amplitudes can be coupled to the atomic variables in such a way that the spurious spontaneous absorption of the ground state is removed. This can be achieved if the $c$-number multiplicative stochastic process that represents the vacuum fluctuations drives only the population of the excited state and is inhibited due to atomic fluctuations for the population of the ground state. From a stochastic description of broad-band squeezed vacuum and collision we have derived the fast and the slow damping rates of the Bloch polarizations. The model of squeezed vacuum fluctuations is simpler from a stochastic theory which requires the doubling of the random process for vacuum fluctuations. We have shown that the noise-induced strong-field resonance fluorescence spectrum for the stochastic model of squeezed vacuum fluctuations and for finite bandwidth collision can be derived. The strong-field resonance fluorescence and other effects can be studied for finite bandwidth squeezed light in the framework of the same formalism.

\section{Acknowledgments}

The authors would like to thank Prof. K. Wódkiewicz for many useful discussions during preparation of the paper. Without his assistance this paper would never have come into existence. 


\section{Appendix}

The functions $Z(\omega)$ and $N(\omega)$ forming the power spectrum are as follows:

$Z(\omega)=E(\omega) Y(\omega)+F(\omega) X(\omega)$,

$N(\omega)=Y^{2}(\omega)+X^{2}(\omega)$,

where

$$
\begin{aligned}
& E(\omega)=2 p B_{1}(\omega)-\omega B_{2}(\omega)+\frac{\Omega_{0}^{2}}{2}, \\
& F(\omega)=2 p B_{2}(\omega)+\omega B_{1}(\omega), \\
& X(\omega)=B_{4}(\omega)+\omega B_{3}(\omega), \\
& Y(\omega)=2 p B_{3}(\omega)+\Omega_{0}^{2}\left[T_{1}(\omega)+B_{1}(\omega)\right]-2 \omega B_{1}(\omega) B_{2}(\omega) .
\end{aligned}
$$

In these expressions we have used the following notations:

$$
\begin{aligned}
& B_{1}(\omega)=p+\operatorname{Re} B_{11}(\omega), \\
& B_{2}(\omega)=\omega+\operatorname{Im} B_{11}(\omega), \\
& T_{1}(\omega)=A|M| \cos \varphi+\operatorname{Re} B_{12}(\omega), \\
& T_{2}(\omega)=A|M| \sin \varphi+\operatorname{Im} B_{12}(\omega), \\
& B_{3}(\omega)=\left(B_{1}(\omega)\right)^{2}-\left[\left(B_{2}(\omega)\right)^{2}+\left(T_{1}(\omega)\right)^{2}+\left(T_{2}(\omega)\right)^{2}\right], \\
& B_{4}(\omega)=\left(B_{2}(\omega)\right)^{2}\left[\Omega_{0}^{2}+4 p B_{1}(\omega)\right]
\end{aligned}
$$

with

$$
\begin{aligned}
& p=\frac{A(2 N+1)}{2} \\
& P=\left(\mathrm{i} \omega+\frac{1}{\tau}\right)\left[\left(\mathrm{i} \omega+\frac{1}{\tau}\right)^{2}+\Omega_{0}^{2}\right] \\
& B_{11}=\frac{a^{2}\left\{\left[\mathrm{i} \omega+(1 / \tau)^{2}\right]+\Omega_{0}^{2} / 2\right\}}{P} \\
& B_{12}=\frac{a^{2} \Omega_{0}^{2}}{2 P}
\end{aligned}
$$

\section{References}

[1] K. Wódkiewicz, Phys. Rev. A 19, 1686 (1979).

[2] G.W. Gardiner, Phys. Rev. Lett. 56, 1917 (1986).

[3] G.W. Gardiner, Quantum Noise, Springer-Verlag, Berlin 1991.

[4] H.J. Carmichael, A.S. Lane, D.F. Walls, Phys. Rev. Lett. 58, 2538 (1987).

[5] A.S. Parkins, C.W. Gardiner, Phys. Rev. A 37, 3867 (1988). 
[6] P.V. Vinh, Ph.D. Thesis, Warsaw University, Warsaw 1998; C.W. Gardiner, A.S. Parkins, Phys. Rev. A 50, 1792 (1994).

[7] H. Ritsche, P. Zoller, Phys. Rev. A 38, 4657 (1988).

[8] E. Kahler, K. Wódkiewicz, J. Mod. Opt. 41, 491 (1994).

[9] P.W. Milonni, Am. J. Phys. 52, 340 (1984).

[10] P.W. Milonni, P.L. Knight, Phys. Rev. A 10, 1096 (1974).

[11] K. Wódkiewicz, Phys. Rev. A 38, 2932 (1988).

[12] M.J. Collett, C.W. Gardiner, Phys. Rev. A 30, 1368 (1984).

[13] K. Wódkiewicz, J.H. Eberly, Phys. Rev. A 32, 992 (1985).

[14] K. Wódkiewicz, N.H. Cong, in: Spectral Line Shapes, Vol. 5, Ossollineum, Wrocław (Poland) 1989, p. 527.

[15] Th. Haslwanter, H. Ritsch, Phys. Rev. A 38, 5652 (1988).

[16] A.A. Rangwala, K. Wódkiewicz, C. Su, Phys. Rev. A 42, 6651 (1990).

[17] K. Wódkiewicz, B.W. Shore, J.H. Eberly, Phys. Rev. A 30, 2390 (1984). 